\title{
Transfer and motivation after cognitive control training for remitted depression in healthy sample
}

Jasmien Vervaeke ${ }^{\mathrm{a}, \mathrm{b}, *}, \mathrm{MSc}$; Kristof Hoorelbeke ${ }^{\mathrm{a}}, \mathrm{PhD}$; Chris Baeken ${ }^{\mathrm{c}, \mathrm{d}, \mathrm{e}}, \mathrm{MD}, \mathrm{PhD}$; Jan Van Looy $^{\mathrm{f}}, \mathrm{PhD}$, \& Ernst H.W. Koster ${ }^{\mathrm{a}}, \mathrm{PhD}$

a: Department of Experimental Clinical and Health Psychology, Henri Dunantlaan 2, Ghent University, Ghent, Belgium

b: imec-mict-Ghent University, Miriam Makebaplein 1, Ghent, Belgium

c: Department of Psychiatry and Medical Psychology, University Hospital Ghent, De Pintelaan 185, Ghent University, Ghent, Belgium

d: Department of Psychiatry, University Hospital Brussels, Brussels, Belgium

e: Ghent Experimental Psychiatry (GHEP) Lab, Ghent University, Ghent, Belgium

f: ML6, Ghent, Belgium

*: Corresponding author: Jasmien.Vervaeke@UGent.be 


\section{Abstract}

Objective: Given that cognitive control deficits following remission from depression form a risk factor for recurrence, new interventions aimed at improving cognitive control such as cognitive control training, are being developed. Previous studies suggest that motivation and engagement can influence effectiveness of cognitive training. As such, we developed a gamified cognitive control training procedure. Before validating this tool in a clinical sample, a convenience sample was used to ensure that gamification did not add any unwanted side effects to the cognitive training procedure.

Materials and methods: This study was preregistered on the Open Science Framework (osf.io/5yacs). Following a baseline assessment, participants were assigned to one of four training conditions, manipulating training task (cognitive control vs. active control) and gamification level (low vs. high). Having performed ten sessions, participants were invited for a post-training assessment. Impact of gamification on cognitive transfer, motivation, and emotional transfer was investigated.

Results: Our results suggest task-specific near cognitive transfer. In line with our hypotheses, gamification level did not affect cognitive transfer while beneficially impacting motivation. Moreover, beneficial effects of gamified cognitive control training were found for selfreported anxiety levels, in absence of effects on the other clinical outcomes.

Conclusion: This study showed that the newly developed gamified cognitive control training procedure yields similar effects as non-gamified cognitive control training. However, the advantage of this newly developed version is that it is more user-friendly, easy-to-use, online, and that its settings maximize user motivation and engagement, potentially enabling a more effective training. 
Keywords: internet intervention, relapse prevention, depression, cognitive control training, gamification, transfer, motivation 


\section{Introduction}

Cognitive remediation (CR) therapy, referring to treatments consisting of systematic exercises for altering neurocognitive abilities (Robertson and Murre 1999) is recently being applied in affective disorders such as major depressive disorder (MDD) (Bowie et al. 2013; Porter et al. 2013). The underlying idea is that depression is characterized by cognitive deficits (Austin et al. 1992), including impaired cognitive control, which frequently remain present following remission from depression (Paelecke-Habermann et al. 2005; Demeyer et al. 2012; Levens and Gotlib 2015). Cognitive control is key for regulating thoughts and goaldirected behavior. Importantly, such deficits may increase cognitive vulnerability for future recurrence of depressive episodes (Gotlib and Joormann 2010; De Raedt and Koster 2010), and existing treatments such as antidepressant medication do not seem to target these cognitive deficits directly (Shilyansky et al. 2016). As such, developing methods that may remediate cognitive impairments in MDD presents a crucial next step.

Although individual cognitive control studies in MDD have produced mixed findings, metaanalyses have shown that there is a small but significant beneficial effect of cognitive training on depressive symptoms (Anaya et al. 2012; Motter et al. 2016). This indicates that cognitive training is of potential interest in depression because of transfer effects on both cognitive and affective functioning. In the CR literature, cognitive transfer of training is considered imperative to obtaining beneficial effects on clinical outcomes. In the next sections, literature regarding cognitive and emotional transfer effects is discussed. Lastly, the role of motivation and engagement is considered.

\section{Cognitive and emotional transfer}

Multiple studies have explored effects of cognitive control training on indicators of cognitive functioning (e.g., cognitive task performance, referred to as cognitive transfer) and 
clinical outcomes (emotional transfer). Within cognitive transfer, we can distinguish near and far transfer effects. Task-specific near cognitive transfer in the current paper refers to training-related performance changes on a task similar to the training task, whereas far cognitive transfer refers to training-related performance changes on a task dissimilar to the training task. Training studies aimed at improving cognitive control in the context of depression have utilized a wide variety of training parameters (see Koster et al. 2017 for a review). Two training paradigms have been dominantly used in the cognitive control training literature: the n-back task (Kirchner 1958; Jaeggi et al. 2003; 2008) and the Paced Auditory Serial Addition Task (PASAT; Gronwall 1977).

Although cognitive transfer has been investigated extensively in healthy samples using the n-back paradigm (Kirchner 1958; Jaeggi et al. 2003), clear transfer effects are oftentimes not found. In particular, demonstrating far cognitive transfer has shown to be a challenging endeavor (which is true for cognitive training in general, see Sala et al. 2019). In this context, several factors have been raised, among which age (Katz et al. 2016), personality (Katz et al. 2016; Studer-Luethi et al. 2012), training or study parameters (Schwaighofer et al. 2015; Foroughi et al. 2016; Dougherty et al. 2016), and motivation (Katz et al. 2016; Jaeggi et al. 2014). In addition, n-back training procedures have yielded limited evidence for emotional transfer effects in the context of depression (for a review, see Koster et al. 2017). That is, as to date emotional transfer effects of the n-back task in the context of depression have only been studied in two studies using a clinical sample. First, Iacoviello and colleagues (2014) compared an emotional n-back training to a neutral n-back training. Emotional transfer effects - in terms of beneficial effects of training on depressive symptomatology were strongest in the emotional n-back training group. However, no differential effects of training condition were found on indicators of cognitive transfer. Given that no active control condition was used, it is impossible to distinguish training effects from effects of time. 
Furthermore, this study included only 21 participants, which may have resulted in a lack of power. Second, Wanmaker and colleagues (2015) compared a dual n-back training to an active control condition using a larger sample size. Despite of demonstrating cognitive transfer effects on an inhibition task, no emotional transfer effects were obtained.

In contrast, studies using the PASAT-based training procedure have yielded more promising clinical findings. At the same time, however, studies investigating cognitive transfer after PASAT-based training are rather scarce and results are mixed. For instance, in remitted depressed (RMD) individuals, task-specific near transfer effects and reduced cognitive complaints have been observed, even at three months follow-up (Hoorelbeke and Koster 2017). Additionally, combining PASAT training with tDCS in a depressed sample improved performance on a 2-back task (Segrave et al. 2014). Here, it is noteworthy that other studies using the PASAT-based training procedure in both clinical and non-clinical samples provided mixed cognitive transfer effects. For instance, Hoorelbeke et al. (2016) observed a trend for improved performance on a dual n-back task following training compared to an active control condition. However, multiple studies failed to demonstrate cognitive transfer of PASAT training on other far transfer measures (e.g., Westerberg et al. 2008; Brehmer et al. 2011; Calkins and Otto 2013, Hoorelbeke et al. 2015; Moshier 2015; Moshier et al. 2015).

Although little is known regarding the cognitive mechanisms underlying the PASAT training procedure, it has shown some effectiveness in reducing depressive symptoms in both MDD and RMD samples. That is, several studies demonstrated beneficial effects of PASATbased training on depressive symptomatology (Siegle et al. 2007; Segrave et al. 2014; Calkins et al. 2015; Hoorelbeke and Koster 2017). In addition, PASAT-based training procedures have shown to positively impact emotion regulation processes, where positive 
effects were observed on depressive rumination (e.g., Siegle et al. 2014; Hoorelbeke and Koster 2017).

Theories explaining how PASAT and dual n-back training may yield beneficial effects are still largely untested. Nevertheless, it has been posed that both tasks recruit the dorsolateral prefrontal cortex (dual n-back: Owen et al. 2005; PASAT: Lazeron et al. 2003), which typically suffers from decreased activation during and after MDD (as inferred from neuropsychological studies such as Austin et al. 1992; Paelecke-Habermann et al. 2005; Demeyer et al. 2012; Levens and Gotlib 2015; and neuroimaging studies such as Baxter et al. 1989; Bench et al. 1992; Drevets 2000). Interestingly, it has been suggested that inhibition of limbic interference while performing the PASAT is crucial for task performance. That is, the PASAT is known to induce frustration and stress (Tombaugh 2006; Siegle et al. 2007), whereas the dual n-back task is not known to induce such feelings. This might be an important differentiator between the two tasks, and explain why the PASAT has shown more clinical effectiveness in the context of depression. In this view, PASAT-based training procedures may train inhibition of limbic interference, which could lead to increased inhibition of stress and associated thoughts. This may explain why beneficial effects of training were previously observed on depressive rumination (e.g., Siegle et al. 2007; 2014; Hoorelbeke and Koster 2017). Furthermore, it also explains partially why emotional n-back training has yielded better results than neutral n-back training (Iacoviello et al. 2014).

Given that there is stronger evidence for the clinical efficacy of PASAT-based training than n-back-based training procedures in the context of depression, we will further investigate cognitive and emotional transfer using this task. Furthermore, in line with previous studies that have demonstrated cognitive transfer following PASAT-based training procedures, we will use a non-adaptive PASAT task to evaluate task-specific near cognitive 
transfer effects (e.g., Hoorelbeke and Koster 2017), whereas far cognitive transfer effects will be assessed using a n-back task (e.g., Segrave et al. 2014; Hoorelbeke et al. 2016).

\section{Motivation, engagement and gamification}

One topic receiving little attention in the cognitive control training literature is engagement and motivation, which may play a key role, given that training procedures usually consist of several sessions, lasting days, weeks or months; and that motivation of the participants is crucial for transfer after CR procedures (Baldwin and Ford 1988; Grossman and Salas 2011; Baldwin et al. 2009). Indeed, motivation has been shown to moderate n-back performance (Katz et al. 2016; Jaeggi et al. 2014) and early findings with the PASAT suggest task engagement is key, with engaged patients (as indexed by pupil dilation) ruminating less and showing less need for treatment (Siegle et al. 2014). In this context, gamification - i.e. adding game elements to non-game contexts (Deterding et al. 2011) - may play an important role in transferability of cognitive training.

Recently, gamification has increasingly been implemented in CR research (for a review, see Lumsden et al. 2016) and has shown beneficial effects (Looyestyn et al. 2017; Khatib et al. 2011; Thom et al. 2012). However, the desired effects of gamification have been absent in some cases (Trushell and Maitland 2005; Parish-Morris et al. 2013; Hawkins et al. 2013; Katz et al. 2014). Particularly regarding CR, gamification often increases motivation but has rarely shown to impact training outcomes (Lumsden et al. 2016; Deveau et al. 2015; Mohammed et al. 2017). Moreover, it has been suggested that adding gamification to a training procedure can negatively affect cognitive transfer. For instance, the impact of several gamification elements in n-back training was examined recently (Katz et al. 2014). When task accuracy was presented visually, performance decreased, resulting in reduced cognitive 
transfer. Gamification was assumed to have distracted from training, causing worse performance and less transfer (Katz et al. 2014).

Although maximizing task engagement seems key to cognitive training procedures, as to date no PASAT-based training studies have investigated effects of gamification on cognitive and emotional transfer. As such, before implementing a modified training in a clinical sample, cognitive transfer effects of gamified PASAT training should be explored.

\section{Aim of the study}

The aim of the current study is to examine the impact of gamification on user experience, cognitive transfer, and emotional transfer following adaptive PASAT training. A cognitive control training platform was developed based on a focus group with remitted depressed patients (Vervaeke et al. 2018). Before testing this platform using a clinical sample, it is undergoing several development steps, to test essential features for usability in interventional research, following an experimental medicine approach (Riddle and Science of Behaviour Change Working Group 2015). Our first aim was to map task-specific near and far cognitive transfer effects using a standardized version of the PASAT and a dual n-back task where we predicted finding both task-specific near and far cognitive transfer (Hypothesis 1 and 2). In addition, we wanted to ensure that gamifying PASAT-based training did not hinder cognitive transfer. In particular, we hoped to find no differences between the high- and lowgamified training tasks in terms of cognitive transfer effects (Hypothesis 3). In contrast, we expected beneficial effects of gamification on user experience, among which self-reported motivation and engagement (Hypothesis 4). Finally, we explored emotional transfer effects of the (gamified) adaptive PASAT training. However, given that a non-selected convenience sample was used, we did not expect to find emotional transfer (Hypothesis 5). 


\section{Method}

\section{Design}

The study was approved by the local ethical committee and pre-registered on the Open Science Framework (osf.io/5yacs) before data collection. Written informed consent was obtained for all participants. The study comprised three phases: baseline assessment, experimental manipulation, and post-assessment. The experimental manipulation consisted of a 2 (training task: cognitive control vs. active control) by 2 (gamification level: high vs. low gamification) design, resulting in four cognitive training conditions. Baseline- and postassessment were identical for all conditions. The training task was manipulated to measure transfer effects. Gamification level was manipulated to obtain knowledge regarding training progress and cognitive transfer. Additionally, the study aimed to test how gamification affected user engagement and motivation.

\section{Sample and data exclusion}

Participants were recruited via a Ghent University research platform, a lab database and social media. Only native Dutch speakers were eligible, due to the language of the training task. Based on a-priori power analysis (see preregistration), 168 participants started the study and were randomly assigned to one of four conditions. The full protocol was completed by 153 participants, losing 15 participants to dropout. Furthermore, six participants failed one or more attention check items while completing baseline- or posttraining questionnaires and were therefore excluded from analyses, but this did not impact the primary pattern of results ${ }^{1}$. Final sample included 147 participants. There were no pre-

\footnotetext{
${ }^{1}$ The results were similar for all analyses except for one. When including these six participants, there was no longer a significant impact of gamification on Expectancy $\left(F(1,148)=3.73 ; p=.055 ; \eta_{p}{ }^{2}=.03\right)$.
} 
existing differences between conditions (see Table 1 and 2). Mean age was 21.4 years and $74.1 \%$ were female, with neither age nor sex differing significantly between conditions (Age: $\left.F(3,143)=1.06, p=.368 ; \operatorname{Sex}: \chi^{2}(3)=0.28, p=.964\right)$.

\section{Material}

Baseline and post-training assessments were run on lab computers with a Windows 7 operating system. Questionnaires and non-adaptive PASAT were administered through a cognitive training platform, developed for this project, on Google Chrome. The training was administered online between baseline- and post-training assessment, using the same platform. The dual n-back task was administered with Inquisit Millisecond Web license, on Internet Explorer.

Training tasks

The experimental manipulation comprised a two-week cognitive training procedure which differed in task content (cognitive control training vs. active control training) and gamification level (high vs. low). Participants completed 10 sessions (15 minutes each) of the assigned task.

The cognitive control training (CCT) task consisted of an adaptive version of the PASAT. During this task, a continuous stream of digits (1-9) was presented and participants had to click the sum of the last two heard digits on a trial-by-trial basis. Cognitive control was trained using an adaptive inter-trial interval (ITI): every four consecutive correct responses, the ITI shortened by $100 \mathrm{~ms}$; following four consecutive incorrect responses, the ITI increased by $100 \mathrm{~ms}$. In addition, each session started with an ITI $500 \mathrm{~ms}$ longer than the median ITI of the previous session. 
Participants receiving active control training (ACT) performed 10 sessions of a low-cognitive load modification of the adaptive PASAT (Hoorelbeke et al. 2016). This task was visually identical to the CCT task, but instructions differed. Participants heard numbers (1-18) and had to click on the corresponding number (speed-of-response training). This task used the same principles of adaptivity, affecting task difficulty within each session and over multiple sessions.

Each of these training tasks existed in two versions: lightly and heavily gamified. For clarity, these will be referred to as low vs. high gamification. The two versions were kept as similar as possible except for gamification elements so that training instructions and mechanisms were identical. Screenshots of both task versions are presented in Figure 1. In the high gamification condition, several elements were added in order to foster a positive user experience, potentially impacting engagement. Visually, the task contained more color(s) and elements, resulting in a more vivid look. The main color theme also varied randomly per training session. Furthermore, session progress was illustrated by a bar in the low gamification version whereas a countdown timer was presented in the high gamification version. In addition, the high gamification version contained motivating messages. These were provided regardless of actual performance in order to foster task engagement and acted as simulated social feedback. The training level was made visible and increased whenever the ITI did, reinforcing the efforts of participants. In both conditions, there was an identical adaptive component, meaning that the pace increased or decreased after four consecutive correct or incorrect responses. While this was not visualized in the low gamification condition, it was in the high gamification version, along with an auditory cue announcing an increase in speed. Upon completing a training session, participants in the high gamification version received a session score and were able to compare this with scores of previous sessions, while this was not the case in the low gamification versions. Both versions provided 
feedback on accuracy and speed. Finally, only in the high gamification versions was there a locked grid at the start of training, in which a cell was unlocked with each session, revealing a motivational quote. Upon completion of the full training procedure (10 sessions), all grid cells were unlocked. Input regarding gamification was collected in a previous stage taking into account the needs and concerns of the users (for a thorough discussion, we refer to Vervaeke et al. 2018).

Cognitive transfer measures

To assess task-specific near transfer effects of the training procedure, the nonadaptive PASAT (Gronwall 1977) was administered at baseline and following completion of the training procedure. The non-adaptive PASAT comprised three blocks containing 60 trials each with a fixed ITI (Block 1: 3000 ms; Block 2: 2000 ms; Block 3: 1500 ms), making the task more difficult each consecutive block. The outcome variable was mean accuracy. In this task, participants continuously responded to the sum of each last two heard digits, similarly to the adaptive PASAT, making this a fitting task-specific near transfer measure.

The dual n-back task (Jaeggi et al. 2010) was used to measure far transfer. This task was used in Hoorelbeke et al. (2016) to measure gains after PASAT-based cognitive training. While the PASAT utilizes primarily attention, processing speed and working memory (Tombaugh 2006), the dual n-back task assesses multiple executive functions as well, with little but some overlap in required processes compared to the PASAT (i.e., inhibition of irrelevant items and updating representations in working memory; Jaeggi et al. 2008), making it a well-suited measure of far transfer.

This task used both auditory and visual stimuli. Participants heard one letter from a subset of eight possible consonants. Simultaneously, a blue square was presented visually in one of eight possible locations. Participants indicated whether there was an auditory match, a 
visual match, or both, with target stimuli presented $\mathrm{N}$ trials back (increasing from two to four). Every level of $\mathrm{N}$ contained three blocks with 20 target trials. The outcome variable was accuracy, expressed as the proportion of hits minus false alarms, halved, and divided by the number of blocks (Jaeggi et al. 2010). The number of hits and false alarms are absolute values. If there are more hits than false alarms, the outcome value will thus be positive. If the number of false alarms is higher, this value will be negative. Higher outcome values reflect better performance.

Indicators of emotional transfer

As secondary outcome measures, several indicators of emotional transfer were assessed. For this purpose, the following three questionnaires were administered in Dutch at baseline and following two weeks of training: Depression Anxiety Stress Scale (DASS; Lovibond and Lovibond 1995; de Beurs et al. 2001), Ruminative Response Scale (RRS; Nolen-Hoeksema and Morrow 1991; Treynor et al. 2003), and the effortful control subscale of the Adult Temperament Questionnaire (ATQ-EC; Rothbart et al. 2000; Hartman and Rothbart 2001) (see supplemental material for more information).

Indicators of motivation

Furthermore, indicators of motivation were included in this study. To check if and how our manipulations impacted the training's credibility, the Credibility/Expectancy Questionnaire (CEQ; Devilly and Borkovec 2000) was administered at baseline- and postassessment, alongside an additional item enquiring whether participants believed themselves to be in a placebo condition (similar to Hoorelbeke and Koster 2017). During post-training assessment, additional questionnaires were administered: the Intrinsic Motivation Inventory (IMI; Ryan 1982; McAuley et al. 1989) (5 subscales: interest/enjoyment, perceived competence, value/usefulness, felt pressure/tension and effort/importance; the subscales 
perceived choice and relatedness were left out for brevity and relevance), the Survey to Evaluate Engagement (SEE; Jacques 1996) and the User Engagement Scale (UES; O'Brien and Toms 2010) (see supplementary material for more information).

\section{Procedure}

Baseline assessments started with general and practical information, followed by questionnaires and the non-adaptive PASAT. Next, participants completed the dual n-back task, followed by instructions regarding the training procedure and online training platform. The baseline assessment lasted between 50 and 60 minutes.

The training itself comprised ten sessions of 15 minutes each. Participants were instructed to complete all sessions within 14 days. Upon completion of the training task, participants could plan their next training session, and e-mail reminders were sent accordingly. Finally, performance feedback was shown.

Post-training assessments were scheduled 11 working days after baseline and started with questionnaires and non-adaptive PASAT, on the same website used for training. Thereafter, the dual n-back task was administered again, followed by debriefing. The posttraining assessment lasted for 45 to 55 minutes. After completing the full procedure, participants received 40 euros.

\section{Data analysis}

Analyses conducted were in accordance with preregistration. However, for analyses on the pre-post data, ANCOVAs were conducted (with pre-test data as covariates) instead of mixed ANOVAs (see Dimitrov and Rumrill 2003). Assumptions were checked and when violated, corrected, resulting in adjusted degrees of freedom. For one participant, the postmeasurement for the dual n-back task was missing. Consequently, this participant was excluded from analyses involving this task, but included for other analyses. Furthermore, due 
to a technical error in the dual n-back task, visual modality responses were not recorded.

Therefore, as outcome measure, the proportion of hits minus false alarms in the auditory modality, averaged over the number of blocks was used, as in the single n-back task (Jaeggi et al. 2010). 


\section{Results}

\section{Training progression}

In line with previous studies (Hoorelbeke et al. 2016; Hoorelbeke and Koster 2017), training progress was analyzed separately for CCT and ACT due to the different nature of the training tasks. Two mixed ANOVAs were used to map progress during training, measured by median ITIs per session. For each ANOVA, time (10 training sessions) and gamification (low vs. high) were included as within- and between-subjects factor, respectively. Results showed significant effects of time for both groups $\left(\mathrm{CCT}: F(4.7,339.4)=74.01, p<.001, \eta_{p}{ }^{2}=.50\right.$; ACT: $\left.F(3.8,271.1)=11.16, p<.001, \eta_{p}{ }^{2}=.13\right)$, indicating that median ITI decreased over sessions, reflecting improvement on the training task as shown in Figure 2. Importantly, gamification level did not impact training progression. In other words, gamification did not hinder performance on $\operatorname{CCT}\left(F(1,72)=0.08, p=.783, \eta_{p}{ }^{2}<.01\right)$ nor $\operatorname{ACT}(F(1,71)=0.47, p$ $\left.=.497, \eta_{p}{ }^{2}<.01\right)$. Interactions were not significant $\left(\mathrm{CCT}: F(4.6,333.4)=0.65, p=.651, \eta_{p}{ }^{2}\right.$ $<.01$; ACT: $\left.F(3.8,269.3)=1.50, p=.206, \eta_{p}^{2}=.02\right)$, suggesting that improvements across gamification levels were similar.

\section{Primary outcome measures}

H1 and H3: Task-specific near cognitive transfer and impact of gamification

To examine whether task-specific near transfer occurred, a 2 (task: CCT vs. ACT) by 2 (gamification: low vs. high) ANCOVA was conducted on accuracy of the non-adaptive PASAT at post-assessment. There was a significant effect of training task but not of gamification, after controlling for baseline scores. Furthermore, the training task by gamification interaction was not significant (Table 3).

Follow-up independent samples $t$-tests revealed no difference between training groups at baseline $(t(145)=0.88, p=.38, d=.14)$ and a significant difference during post- 
assessment $(t(128.3)=15.49, p=.007, d=2.56)$. All groups improved from baseline to postassessment; however, the CCT-group improved more (from $38.6 \%$ to $84.3 \%$; compared to ACT: from $40.2 \%$ to $54.7 \%$ ), indicating a task-specific near transfer effect of CCT.

$\mathrm{H} 2$ and $\mathrm{H} 3$ : Far cognitive transfer and impact of gamification

To check for far transfer, another $2 \times 2$ ANCOVA was conducted, using performance on the dual N-back task. Neither training task, gamification or their interaction yielded significant effects (Table 3). Hence, there was no evidence for training-related far transfer effects. However, it is possible that participants were performing differently in the visual modality of the task and that transfer occurred there. Due to the data loss in this modality, we will refrain from making strong claims regarding far transfer.

\section{Secondary outcome measures}

H4: Impact of gamification level on motivation

At baseline, conditions were similar in terms of credibility, expectancy, and belief to be in a placebo condition. At post-assessment however, motivational differences were found (Table 4). Conducting 2x2 ANOVAs (Table 5), the post-training scores of the SEE, IMI interest/enjoyment and perceived competence were significantly higher in the high gamification condition. Furthermore, values for the UES and IMI value/usefulness were marginally higher in this condition. Interestingly, there was no effect of gamification on the IMI effort/importance and felt pressure/tension. None of the interactions were significant. These findings suggest that the gamification manipulation was able to increase motivation and engagement, and did not result in one task version to be perceived as more difficult or stressful. However, pre-existing differences is an alternative assertion that might explain these results too (but were absent in terms of credibility and expectancy of the intervention, see Table 2). 
Additionally, the scores for IMI value/usefulness were significantly higher in the CCT condition. This might be reflecting that the ACT condition was perceived as less beneficial. This might also explain the findings at post-assessment regarding the CEQ and the placebo check item. Two 2x2 ANCOVAs revealed a significant effect of training task and gamification on the post-scores of the two CEQ subscales (Table 3) but no interaction. Follow-up independent samples $t$-tests showed higher scores for high gamification conditions and CCT conditions, where all differences were significant (CEQ Credibility: training task: $t(145)=4.96, p<.001, d=.82$; gamification: $t(145)=3.14, p=.002, d=.52 ;$ CEQ Expectancy: training task: $t(136.7)=2.60, p=.010, d=.43$; gamification: $t(140.0)=2.16, p$ $=.033, d=.36)$. A binary logistic regression showed that training task helped predict participants' response on the placebo check item, while gamification did not (Table 3). Participants in the ACT condition were 3.7 times more likely to report believing to be in a placebo condition.

H5: Emotional transfer

Emotional transfer effects were assessed using 2x2 ANCOVAs (Table 3). There was a main effect of gamification on the attentional control component of the ATQ-EC, indicating higher scores in the high gamification conditions. The training task by gamification interaction was significant for the anxiety component of the DASS. Post-hoc testing revealed significant differences, while controlling for baseline scores. In the CCT group, high gamified training led to lower anxiety scores $\left(F(1,142)=4.69, p=.032, \eta_{p}{ }^{2}=.03\right)$, while this was not the case in the ACT group $\left(F(1,142)=1.56, p=.213, \eta_{p}{ }^{2}=.01\right)$. Additionally, within the high gamification group, CCT led to lower anxiety scores than $\mathrm{ACT}(F(1,142)=$ 4.16, $p=.043, \eta_{p}{ }^{2}=.03$ ), and this difference was absent in the low gamification conditions $\left(F(1,142)=1.85, p=.176, \eta_{p}{ }^{2}=.01\right)$. Although this seems to indicate that some emotional 
transfer occurred, estimates of effect sizes were small and there was substantial variability (Figure 3). All other analyses regarding emotional transfer were non-significant.

Dropout analysis

Of the 168 participants that completed the baseline assessment, 15 dropped out. Out of those 15 , two did not start the training. The remaining 13 participants were divided evenly over conditions, suggesting no significant differences of high vs. low gamification on dropout rates: $\chi^{2}(1)=.008 ; p=.685$. 


\section{Discussion}

This study examined the impact of a gamified PASAT-based training procedure on task-specific near and far cognitive transfer, user experience, and emotional transfer, in a healthy sample. Our aim was to ensure that gamification did not impair cognitive transfer while increasing motivation, before implementing training in a clinical context. Training task (cognitive control training (CCT) vs. active control condition) and gamification level (low vs. high) were manipulated, resulting in four between-subjects conditions. We found that, irrespective of training task or gamification level, participants improved during training. Furthermore, in the CCT condition, there was clear evidence for task-specific near cognitive transfer. Far transfer did not appear to occur, but due to partial data loss, this cannot be confirmed or denied using the available data. The motivation and engagement scores were higher in the high gamification version of the training task, while performance was not affected. However, no effects of gamification were observed with regard to dropout. Lastly, as expected for a healthy sample no evidence was found for emotional transfer on all measures, except for anxiety scores, which were lower in the highly gamified CCT group in comparison to other groups. These findings are discussed below.

The findings show that CCT elicits a task-specific near transfer effect. However, for CCT to be effective in a clinical context, far transfer should also occur. Even if there was no data loss, absence of far transfer effects would have been unsurprising and might have been due to the chosen sample which consisted of healthy individuals (where far transfer might hardly occur, see Sala et al. 2019 for a second-order meta-analysis), with little variation in cognitive control. Sampling from restricted ranges of the total population, for instance students, can bias results due to reduced variability, making effect sizes smaller (Bobko et al. 2001; Fritz et al. 2012). For instance, Hoorelbeke et al. (2016) observed a tendency for improved task performance on the dual n-back task in a convenience sample following 
PASAT-based training. However, currently, it is not possible to conclude whether or not far transfer occurred.

Importantly, the gamification manipulation seemed to result in a more positive user experience and higher motivation without altering the transfer effects. As such, there appeared not to be any downsides to including the gamification elements used in the current study. This means that adding this type of gamification might help prevent dropout in this training, without impairing cognitive transfer. In the current sample we did not observe any direct effects on dropout but this could change when training is applied to the intended users that frequently experience motivational impairments. Emotional transfer was only seen in anxiety scores, as measured with the DASS. Scores were significantly lower at post-training assessment for the highly gamified CCT group compared to other groups, while controlling for baseline scores. This means that implementing gamification elements in CCT might lead to a more effective training, as clinical outcome measures are affected. However, caution with interpretation of this finding is advised since we observed considerable individual variability. None of the other emotional transfer variables showed any effect, as was expected in the current healthy sample.

Overall, the current study is encouraging for implementing gamified CCT in a clinical sample. However, there are some limitations to this study as well. First, due to data loss in the dual n-back task, we cannot interpret the far transfer effects readily. We did not find transfer for the auditory modality. The lack of this effect might mean that the training was simply not effective in terms of cognitive transfer. However, other explanations are viable too. For instance, it might be possible that there was a transfer effect in the visual modality, or when considering both modalities. Another possibility might be related to the sample. We chose a healthy convenience sample, with unimpaired cognitive control functions, making improvement less probable. Second, there was limited evidence for emotional transfer. That 
is, we only observed improvements in anxiety levels. As such, it might be that the training was not effective. However, if this were the case, transfer to anxiety scores are not likely to occur. Third, participants experienced the training as being unpleasant, frustrating, and stressful. Most completed the protocol merely to receive the incentive. As such, it might be hard to motivate patients to complete all the sessions, as they will not receive a monetary incentive. However, a focus on relapse prevention could be motivating enough for this target population. Therefore, thorough psycho-education will be included.

In conclusion, this study provides an essential preparatory step for gamified CCT. There seem to be similar effects with previous versions, and the gamification did not interfere with training effects. We found no reason not to implement this new version of CCT in atrisk and clinical samples, which forms the next step. 


\section{Acknowledgments}

The authors wish to thank Sara De Witte and Alexander Goldhoorn for their help with data collection.

\section{Author Disclosure Statement}

This research was supported by an Applied Biomedical (TBM) grant of the Agency for Innovation through Science and Technology (IWT), part of the Research FoundationFlanders (FWO), awarded to the PrevenD project (B/14730/01). KH is a Postdoctoral Fellow of the FWO (FWO.3EO.2018.0031.01). No competing financial interests exist.

\section{Conflict of interest}

On behalf of all authors, the corresponding author states that there is no conflict of interest. 
References

Anaya, C., Aran, A. M., Ayuso-Mateos, J. L., Wykes, T., Vieta, E., \& Scott, J. (2012). A systematic review of cognitive remediation for schizo-affective and affective disorders. Journal of affective disorders, 142(1-3), 13-21.

Austin, M. P., Ross, M., Murray, C., O'Cárroll, R. E., Ebmeier, K. P., \& Goodwin, G. M. (1992). Cognitive function in major depression. Journal of affective disorders, 25(1), 21-29.

Baldwin, T. T., \& Ford, J. K. (1988). Transfer of training: A review and directions for future research. Personnel psychology, 41(1), 63-105.

Baldwin, T. T., Ford, J. K., \& Blume, B. D. (2009). Transfer of training 1988-2008: an updated review and agenda for future research. International review of industrial and organizational psychology, 24(1), 41-70.

Baxter, L. R., Schwartz, J. M., Phelps, M. E., Mazziotta, J. C., Guze, B. H., Selin, C. E., ... \& Sumida, R. M. (1989). Reduction of prefrontal cortex glucose metabolism common to three types of depression. Archives of general psychiatry, 46(3), 243-250.

Bench, C. J., Friston, K. J., Brown, R. G., Scott, L. C., Frackowiak, R. S., \& Dolan, R. J. (1992). The anatomy of melancholia-focal abnormalities of cerebral blood flow in major depression. Psychological medicine, 22(3), 607-615.

Bobko, P., Roth, P. L., \& Bobko, C. (2001). Correcting the effect size of d for range restriction and unreliability. Organizational Research Methods, 4(1), 46-61.

Bowie, C. R., Gupta, M., Holshausen, K., Jokic, R., Best, M., \& Milev, R. (2013). Cognitive remediation for treatment-resistant depression: effects on cognition and functioning and the role of online homework. The Journal of nervous and mental disease, 201(8), 680-685.

Brehmer, Y., Rieckmann, A., Bellander, M., Westerberg, H., Fischer, H., \& Bäckman, L. (2011). Neural correlates of training-related working-memory gains in old age. Neuroimage, 58(4), 1110-1120.

Calkins, A. W., McMorran, K. E., Siegle, G. J., \& Otto, M. W. (2015). The effects of computerized cognitive control training on community adults with depressed mood. Behavioural and cognitive psychotherapy, 43(5), 578-589.

Calkins, A. W., \& Otto, M. W. (2013). Testing the Boundaries of Computerized Cognitive Control Training on Symptoms of Obsessive Compulsive Disorder. Cognitive Therapy and Research, 37, 587-594. doi: 10.1007/s10608-012-9496-x

de Beurs, E., Van Dyck, R., Marquenie, L. A., Lange, A., \& Blonk, R. W. (2001). De DASS: een vragenlijst voor het meten van depressie, angst en stress. Gedragstherapie, 34(1), 35-54.

Demeyer, I., De Lissnyder, E., Koster, E. H., \& De Raedt, R. (2012). Rumination mediates the relationship between impaired cognitive control for emotional information and 
depressive symptoms: A prospective study in remitted depressed adults. Behaviour research and therapy, 50(5), 292-297.

De Raedt, R., \& Koster, E. H. (2010). Understanding vulnerability for depression from a cognitive neuroscience perspective: A reappraisal of attentional factors and a new conceptual framework. Cognitive, Affective, \& Behavioral Neuroscience, 10(1), 5070 .

Deterding, S., Dixon, D., Khaled, R., \& Nacke, L. (2011, September). From game design elements to gamefulness: defining gamification. In Proceedings of the 15th international academic MindTrek conference: Envisioning future media environments (pp. 9-15). ACM.

Deveau, J., Jaeggi, S. M., Zordan, V., Phung, C., \& Seitz, A. R. (2015). How to build better memory training games. Frontiers in systems neuroscience, 8, 243.

Devilly, G. J., \& Borkovec, T. D. (2000). Psychometric properties of the credibility/expectancy questionnaire. Journal of behavior therapy and experimental psychiatry, 31(2), 73-86.

Dimitrov, D. M., \& Rumrill Jr, P. D. (2003). Pretest-posttest designs and measurement of change. Work, 20(2), 159-165.

Dougherty, M. R., Hamovitz, T., \& Tidwell, J. W. (2016). Reevaluating the effectiveness of n-back training on transfer through the Bayesian lens: Support for the null. Psychonomic Bulletin \& Review, 23(1), 306-316.

Drevets, W. C. (2000). Neuroimaging studies of mood disorders. Biological psychiatry, $48(8), 813-829$.

Foroughi, C. K., Monfort, S. S., Paczynski, M., McKnight, P. E., \& Greenwood, P. M. (2016). Placebo effects in cognitive training. Proceedings of the national Academy of Sciences, 201601243.

Fritz, C. O., Morris, P. E., \& Richler, J. J. (2012). Effect size estimates: current use, calculations, and interpretation. Journal of experimental psychology: General, 141(1), 2.

Gotlib, I. H., \& Joormann, J. (2010). Cognition and depression: current status and future directions. Annual review of clinical psychology, 6, 285-312.

Gronwall, D. M. A. (1977). Paced auditory serial-addition task: a measure of recovery from concussion. Perceptual and motor skills, 44(2), 367-373.

Grossman, R., \& Salas, E. (2011). The transfer of training: what really matters. International Journal of Training and Development, 15(2), 103-120.

Hartman, C. A., \& Rothbart, M. K. (2001). Dutch translation of the adult temperament questionnaire. Groningen: Department of Psychiatry, Internal publication ARIADNE research group.

Hawkins, G. E., Rae, B., Nesbitt, K. V., \& Brown, S. D. (2013). Gamelike features might not improve data. Behavior research methods, 45(2), 301-318. 
Hoorelbeke, K., \& Koster, E. H. (2017). Internet-delivered cognitive control training as a preventive intervention for remitted depressed patients: Evidence from a doubleblind randomized controlled trial study. Journal of consulting and clinical psychology, 85(2), 135.

Hoorelbeke, K., Koster, E. H., Demeyer, I., Loeys, T., \& Vanderhasselt, M. A. (2016). Effects of cognitive control training on the dynamics of (mal) adaptive emotion regulation in daily life. Emotion, 16(7), 945.

Hoorelbeke, K., Koster, E. H., Vanderhasselt, M. A., Callewaert, S., \& Demeyer, I. (2015). The influence of cognitive control training on stress reactivity and rumination in response to a lab stressor and naturalistic stress. Behaviour research and therapy, 69, 1-10.

Iacoviello, B., Huryk, K., Alvarez, E., Collins, K., Murrough, J., Iosifescu, D., \& Charney, D. (2014). Cognitive-Emotional Training as an Intervention for Major Depressive Disorder. Biological Psychiatry, 75(9), 119S-119S.

Jacques, R. D. (1996). The nature of engagement and its role in hypermedia evaluation and design (Doctoral dissertation, South Bank University).

Jaeggi, S. M., Buschkuehl, M., Jonides, J., \& Perrig, W.J. (2008). Improving fluid intelligence with training on working memory. Proceedings of the National Academy of Sciences, 105(19), 6829-6833. doi: 10.1073/pnas.0801268105

Jaeggi, S. M., Buschkuehl, M., Shah, P., \& Jonides, J. (2014). The role of individual differences in cognitive training and transfer. Memory \& cognition, 42(3), 464-480.

Jaeggi, S. M., Seewer, R., Nirkko, A. C., Eckstein, D., Schroth, G., Groner, R., \& Gutbrod, K. (2003). Does excessive memory load attenuate activation in the prefrontal cortex? Load-dependent processing in single and dual tasks: functional magnetic resonance imaging study. NeuroImage, 19(2), 210-225.

Jaeggi, S. M., Studer-Luethi, B., Buschkuehl, M., Su, Y. F., Jonides, J., \& Perrig, W. J. (2010). The relationship between n-back performance and matrix reasoningimplications for training and transfer. Intelligence, 38(6), 625-635.

Katz, B., Jaeggi, S., Buschkuehl, M., Stegman, A., \& Shah, P. (2014). Differential effect of motivational features on training improvements in school-based cognitive training. Frontiers in human neuroscience, 8, 242.

Katz, B., Jones, M. R., Shah, P., Buschkuehl, M., \& Jaeggi, S. M. (2016). Individual differences and motivational effects. In Cognitive Training (pp. 157-166). Springer, Cham.

Khatib, F., DiMaio, F., Cooper, S., Kazmierczyk, M., Gilski, M., Krzywda, S., ... \& Jaskolski, M. (2011). Crystal structure of a monomeric retroviral protease solved by protein folding game players. Nature structural \& molecular biology, 18(10), 1175.

Kirchner, W. K. (1958). Age differences in short-term retention of rapidly changing information. Journal of experimental psychology, 55(4), 352. 
Koster, E. H. W., Hoorelbeke, K., Onraedt, T., Owens, M., \& Derakshan, N. (2017).

Cognitive control interventions for depression: a systematic review of findings from training studies. Clinical psychology review, 53, 79-92. doi:

10.1016/j.cpr.2017.02.002

Lazeron, R. H., Rombouts, S. A., deSonneville, L., Barkhof, F., \& Scheltens, P. (2003). A paced visual serial addition test for fMRI. Journal of Neurological Science, 213, 29 34.

Levens, S. M., \& Gotlib, I. H. (2015). Updating emotional content in recovered depressed individuals: Evaluating deficits in emotion processing following a depressive episode. Journal of behavior therapy and experimental psychiatry, 48, 156-163.

Looyestyn, J., Kernot, J., Boshoff, K., Ryan, J., Edney, S., \& Maher, C. (2017). Does gamification increase engagement with online programs? A systematic review. PloS one, 12(3), e0173403.

Lovibond, P. F., \& Lovibond, S. H. (1995). The structure of negative emotional states: Comparison of the Depression Anxiety Stress Scales (DASS) with the Beck Depression and Anxiety Inventories. Behaviour research and therapy, 33(3), 335343.

Lumsden, J., Edwards, E. A., Lawrence, N. S., Coyle, D., \& Munafò, M. R. (2016). Gamification of cognitive assessment and cognitive training: a systematic review of applications and efficacy. JMIR serious games, 4(2).

McAuley, E., Duncan, T., \& Tammen, V. V. (1989). Psychometric properties of the Intrinsic Motivation Inventory in a competitive sport setting: A confirmatory factor analysis. Research quarterly for exercise and sport, 60(1), 48-58.

Mohammed, S., Flores, L., Deveau, J., Hoffing, R. C., Phung, C., Parlett, C. M., ... \& Zordan, V. (2017). The benefits and challenges of implementing motivational features to boost cognitive training outcome. Journal of Cognitive Enhancement, 1(4), 491-507.

Moshier, S. J. (2015). Cognitive control training as an adjunct to behavioral activation therapy in the treatment of depression. Boston University.

Moshier, S. J., Molokotos, E. K., Stein, A. T., \& Otto, M. W. (2015). Assessing the effects of depressed mood and cognitive control training on memory confidence and accuracy following repeated checking. International Journal of Cognitive Therapy, 8(3), 206221.

Motter, J. N., Pimontel, M. A., Rindskopf, D., Devanand, D. P., Doraiswamy, P. M., \& Sneed, J. R. (2016). Computerized cognitive training and functional recovery in major depressive disorder: a meta-analysis. Journal of Affective Disorders, 189, 184191.

Nolen-Hoeksema, S., \& Morrow, J. (1991). A prospective study of depression and posttraumatic stress symptoms after a natural disaster: the 1989 Loma Prieta Earthquake. Journal of personality and social psychology, 61(1), 115. 
O'Brien, H. L., \& Toms, E. G. (2010). The development and evaluation of a survey to measure user engagement. Journal of the American Society for Information Science and Technology, 61(1), 50-69.

Owen, A. M., McMillan, K. M., Laird, A. R., \& Bullmore, E. (2005). N-back working memory paradigm: A meta-analysis of normative functional neuroimaging studies. Human Brain Mapping, 25, 46-59. doi: 10.1002/hbm.20131

Paelecke-Habermann, Y., Pohl, J., \& Leplow, B. (2005). Attention and executive functions in remitted major depression patients. Journal of affective disorders, 89(1-3), 125-135.

Parish-Morris, J., Mahajan, N., Hirsh- Pasek, K., Golinkoff, R. M., \& Collins, M. F. (2013). Once upon a time: Parent-child dialogue and storybook reading in the electronic era. Mind, Brain, and Education, 7(3), 200-211.

Porter, R. J., Bowie, C. R., Jordan, J., \& Malhi, G. S. (2013). Cognitive remediation as a treatment for major depression: a rationale, review of evidence and recommendations for future research. Australian \& New Zealand Journal of Psychiatry, 47(12), 1165-1175.

Riddle, M., \& Science of Behavior Change Working Group. (2015). News from the NIH: using an experimental medicine approach to facilitate translational research.

Robertson, I. H., \& Murre, J. M. (1999). Rehabilitation of brain damage: Brain plasticity and principles of guided recovery. Psychological bulletin, 125(5), 544.

Rothbart, M. K., Ahadi, S. A., \& Evans, D. E. (2000). Temperament and personality: origins and outcomes. Journal of personality and social psychology, 78(1), 122.

Ryan, R. M. (1982). Control and information in the intrapersonal sphere: An extension of cognitive evaluation theory. Journal of personality and social psychology, 43(3), 450.

Sala, G., Aksayli, N. D., Tatlidil, K. S., Tatsumi, T., Gondo, Y., \& Gobet, F. (2019). Near and far transfer in cognitive training: A second-order meta-analysis. Fifth version of the preprint.

Schwaighofer, M., Fischer, F., \& Bühner, M. (2015). Does working memory training transfer? A meta-analysis including training conditions as moderators. Educational Psychologist, 50(2), 138-166.

Segrave, R. A., Arnold, S., Hoy, K., \& Fitzgerald, P. B. (2014). Concurrent cognitive control training augments the antidepressant efficacy of tDCS: A pilot study. Brain Stimulation, 7(2), 325-331. doi: 10.1016/j.brs.2013.12.008

Shilyansky, C., Williams, L. M., Gyurak, A., Harris, A., Usherwood, T., \& Etkin, A. (2016). Effect of antidepressant treatment on cognitive impairments associated with depression: A randomised longitudinal study. The Lancet Psychiatry. doi: 10K1016+S2215-0366(16)00012-2 
Siegle, G. J., Ghinassi, F., \& Thase, M. E. (2007). Neurobehavioral therapies in the 21 st century: Summary of an emerging field and an extended example of cognitive control training for depression. Cognitive Therapy and Research, 31(2), 235-262.

Siegle, G. J., Price, R. B., Jones, N. P., Ghinassi, F., Painter, T., \& Thase, M. E. (2014). You gotta work at it: Pupillary indices of task focus are prognostic for response to a neurocognitive intervention for rumination in depression. Clinical Psychological Science, 2(4), 455-471.

Studer-Luethi, B., Jaeggi, S. M., Buschkuehl, M., \& Perrig, W. J. (2012). Influence of neuroticism and conscientiousness on working memory training outcome. Personality and Individual Differences, 53(1), 44-49.

Thom, J., Millen, D., \& DiMicco, J. (2012). Removing gamification from an enterprise SNS. In Proceedings of the acm 2012 conference on computer supported cooperative work (pp. 1067-1070). ACM.

Tombaugh, T.N. (2006). A comprehensive review of the Paced Auditory Serial Addition Test (PASAT). Archives of Clinical Neuropsychology, 21, 53-76. doi: 10.1016/j.acn.2005.05.006

Treynor, W., Gonzalez, R., \& Nolen-Hoeksema, S. (2003). Rumination reconsidered: A psychometric analysis. Cognitive therapy and research, 27(3), 247-259.

Trushell, J., \& Maitland, A. (2005). Primary pupils' recall of interactive storybooks on CDROM: inconsiderate interactive features and forgetting. British Journal of Educational Technology, 36(1), 57-66.

Vervaeke, J., Van Looy, J., Hoorelbeke, K., Baeken, C., \& Koster, E.W.H. (2018). Gamified Cognitive Control Training for Remitted Depressed Individuals: User Requirements Analysis. JMIR serious games, 6(2).

Wanmaker, S., Geraerts, E., \& Franken, I. H. A. (2015). A working memory training to decrease rumination in depressed and anxious individuals: A double-blind randomized controlled trial. Journal of Affective Disorders, 175, 310-319. doi: 10.1016/j.jad.2014.12.027

Westerberg, H., Brehmer, Y., D’Hondt, N., Söderman, D., \& Bäckman, L. (2008). Computerized training of working memory: A controlled, randomized trial. In Poster presented at the meeting of the Cognitive Neuroscience Society, San Francisco, $C A$. 
Address correspondence to:

Jasmien Vervaeke

Department of Experimental Clinical and Health Psychology

Ghent University

Henri Dunantlaan 2

9000 Ghent - Belgium

E-mail: Jasmien.Vervaeke@UGent.be 
Supplemental material - Extra information about questionnaires

S1: Emotional transfer questionnaires

Symptoms of depression, anxiety and stress were assessed by the DASS (range 0 126). Each subscale consisted of 14 items and has recommended clinical cutoffs. Rumination was measured using the 22-item RRS (range 22 - 88). Five items form the subscale brooding, or maladaptive rumination. Reflection refers to purposeful problem-solving strategies and was assessed through five items. Furthermore, 12 items were depression-related. For both questionnaires, higher scores indicated more symptoms or rumination. Effortful control is defined as the ability to suppress a dominant response and choosing a non-dominant one. This was measured by the subscale ATQ-EC which consisted of attentional control (five items), inhibitory control (seven items) and activation control (seven items), measuring capacity to focus and shift attention, capacity to suppress inappropriate behavior and capacity to perform an action when one wants to avoid this, respectively. Each item was scored between one and seven and for each of the three components, a mean score was calculated.

Cronbach's alphas were calculated for each questionnaire on first administration. All were deemed acceptable: $0.95,0.90$ and 0.80 for the DASS, RRS and the ATQ-EC, respectively. Given the interesting aspects of certain subscales, these were also examined separately: depressive symptomatology (0.93), anxiety (0.87) and stress (0.90) for the DASS, maladaptive brooding (0.73) and reflection (0.78) for the RRS, and attentional control (0.62), activation control (0.68) and inhibitory control (0.62) for the ATQ-EC. 


\section{S2: Indicators of motivation}

The IMI assessed the subjective experience and we selected five subscales: interest/enjoyment (7 items), perceived competence (6 items), effort/importance (5 items), value/usefulness (7 items) and felt pressure/tension (5 items). Each of the subscales can be used independently and the subscale interest/enjoyment is considered to be a measure of intrinsic motivation. In analyses, subscale averages are used (so each subscale ranges from 1 to 7). The SEE consists of 14 items on a 5-point scale (range 14-70) and one optional openended item asking for additional comments. It is specifically used for capturing engagement with software, consists of seven attributes: attention, time, motivation, needs, control, attitudes and engagement, and has pre-defined levels of engagement based on the sum. Lastly, the UES also measures engagement, but more specifically in an interactive context and uses sum scores. It contains 31 items (range 31 - 155), divided over 6 factors: focused attention (7 items), perceived usability ( 8 items), aesthetics (5 items), endurability (5 items), novelty (3 items) and felt involvement (3 items).

Cronbach's alphas were calculated for each motivational questionnaire at postassessment. All were deemed acceptable: 0.88, 0.86 and 0.87 for the CEQ, SEE and the UES, respectively. As certain subscales are particularly interesting, their reliability was calculated as well. For the IMI, the subscales interest/enjoyment, perceived competence, effort/importance, value/usefulness and felt pressure/tension all had acceptable reliability, even with just a few items: $0.89 ; 0.92 ; 0.79 ; 0.94$ and 0.79 respectively. 